\title{
FACTORES DE RIESGO ASOCIADOS A SEPSIS NEONATAL TEMPRANA EN PREMATUROS DE UN HOSPITAL NACIONAL DOCENTE MADRE NIÑO, 2017
}

\author{
RISK FACTORS ASSOCIATED WITH EARLY NEONATAL SEPSIS IN PRETERM INFANTS OF THE NATIONAL \\ CHILD MOTHER TEACHING HOSPITAL, 2017 \\ Gretzelle Burga-Montoya ${ }^{1, a}$, Consuelo Luna-Muñoz ${ }^{2,3, a, b}$, Lucy Correa López ${ }^{3}$
}

\begin{abstract}
RESUMEN
Introducción: La sepsis neonatal es una de las enfermedades más importantes de nuestro País, es necesario conocer sus factores de riesgo. Objetivo: Determinar los factores asociados a sepsis neonatal temprana en prematuros del Hospital Nacional Docente Madre Niño San Bartolomé en el año 2017. Métodos: Estudio de casos y controles, observacional, analítico de corte transversal y retrospectivo. La población consta de 210 recién nacidos prematuros del Hospital Nacional Docente Madre Niño San Bartolomé, año 2017. La variable dependiente fue la sepsis neonatal temprana. Se trabajó con un tamaño muestral de 62 casos y 124 controles (ratio 1:2).Los datos se obtuvieron de las historias clínicas. Para la estadística inferencial se utilizó el análisis bivariado para hallar los Odds Ratio con sus respectivos intervalos de confianza al $95 \%$. Se consideró $p<0,05$ como estadísticamente significativo. Resultados: De los 186 pacientes estudiados, 53,8\% fueron del sexo femenino. Los factores asociados a sepsis neonatal temprana fueron: bajo peso al nacer -ORa: 4,031 (IC95\%: 1,743-9,318); edad de 35 a más -ORa: 2,729 (IC95\%: 1,266-5,88); menos de 6 controles prenatales -ORa: 3,315 (IC95\%: 1,452-7,567); infección del tracto urinario en el 3er trimestre -ORa: 2.947 (IC95\%: 1,063-8,174); líquido meconial -ORa: 5,822 (IC95\%: 1,92-17,653) y ruptura prematura de membranas -ORa: 2.789 (IC95\%: 1,035-7,511). Discusión: Se ha encontrado asociación significativa entre sepsis neonatal temprana y bajo peso al nacer, edad de 35 a más, menos de 6 controles prenatales, infección del tracto urinario en el 3er trimestre, líquido meconial y ruptura prematura de membranas.
\end{abstract}

Palabras clave: Sepsis neonatal; Recien nacido prematuro; Enfermedades del prematuro; Líquido amniótico. (fuente: DeCS BIREME)

\begin{abstract}
Introduction: Neonatal sepsis is one of the most important diseases of our country, it is necessary to know its risk factors in our country. Objetive: To determine the risk factors associated with early neonatal sepsis in preterm infants of the National Mother Teaching Hospital San Bartolomé in 2017. Methods: Case-control, observational, analytical, cross-sectional and retrospective study. will work with medical records. The dependent variable was early neonatal sepsis. We worked with a sample size of 62 cases and 124 controls (ratio 1:2). The data was obtained from the medical records. For inferential statistics, bivariate analysis was used to find the Odds Ratio with their respective $95 \%$ confidence intervals. Values $p<0,05$ wereconsidered statistically significant. Results: Of the 186 patients studied, $53,8 \%$ were female. The factors associated with early neonatal sepsis were: low birth weight -ORa: 4,031 (95\% Cl: 1,743-9.318); age 35 to over -ORa: 2,729 (95\% Cl: 1,266-5,88); less than 6 prenatal controls -ORa: 3,315 (IC95\%: 1,452-7,567); urinary tract infection (UTI) in the 3rd quarter-ORa: 2.947 (95\% Cl: 1,063-8,174); meconial fluid -ORa: $5,822(95 \% \mathrm{Cl}: 1,92-17,653)$ and premature rupture of membranes -ORa: $2,789(95 \% \mathrm{Cl}$ : 1,035-7,511). Discussion: A significant association has been found between early neonatal sepsis and low birth weight, age 35 to more, fewer than 6 prenatal controls, UTI in the third trimester, meconium fluid and premature rupture of membranes.
\end{abstract}

Keywords: Neonatal sepsis; Premature newborn; Diseases of prematurity; Amniotic fluid.(source: MeSH NLM)

1 Centro de Salud Pichanaqui, Pichanaqui, Chanchamayo, Junín-Perú.

2 Hospital Nacional Edgardo Rebagliati Martins - EsSalud, Lima-Perú.

3 Instituto de Investigación en Ciencias Biomédicas, Universidad Ricardo Palma, Lima - Perú.

a Médico cirujano.

b Especialista en pediatría.

Citar como: Gretzelle Burga-Montoya, Consuelo Luna-Muñoz, Jhony A. De La Cruz-Vargas. Factores de riesgo asociados a sepsis neonatal temprana en prematuros de un hospital Nacional Docente Madre Niño, 2017. Rev. Fac. Med. Hum. Julio 2019; 19(3):35-42. DOI 10.25176/RFMH.v19i3.2165 


\section{INTRODUCCIÓN}

La sepsis es la disfunción orgánica causada por una respuesta anómala del huésped a la infección, amplificada significativamente por factores endógenos, con alta morbilidad y mortalidad, y representa un desafío para los servicios de neonatología a nivel mundial ${ }^{1,2}$.

Dependiendo de la edad de inicio de la enfermedad, la sepsis neonatal se divide en sepsis neonatal temprana o sepsis de inicio tardío. La sepsis neonatal temprana (SNT) se debe principalmente a organismos adquiridos antes y durante el parto (o infección fetal materna) y la sepsis de inicio tardío (SIT) a organismos adquiridos después del parto (fuentes nosocomiales o comunitarias). Sin embargo, hay poco consenso sobre qué límites de edad se aplican, con una aparición temprana que varía de 48 horas a 7 días después del parto ${ }^{3}$.

En las últimas dos décadas, se ha demostrado un progreso notable en la reducción de muertes maternas e infantiles, y la salud neonatal forma parte de la "agenda inconclusa". El mundo está experimentando un aumento en la proporción de muertes en menores de cinco años que ocurren en el período neonatal ${ }^{4}$. Sin embargo, a pesar de que las muertes neonatales son prevenibles, se concentran en los países más pobres del mundo ${ }^{4}$, y el $85 \%$ se produjeron en países de ingresos bajos y medios (PIMB), a pesar de que albergan solo al $62 \%$ de los recién nacidos del mundo ${ }^{4,5}$.

Globalmente, el $15 \%$ de las muertes neonatales son causadas por la sepsis neonatal y es una preocupación importante en los países de ingresos bajos y medianos ${ }^{4}$.

Además, también se asocia con un aumento de los costos médicos, una estadía hospitalaria prolongada y resultados del neurodesarrollo a largo plazo potencialmente deficientes. De los recién nacidos (RN) que sobreviven, aproximadamente un cuarto tienen secuelas neurológicas significativas como consecuencia de la afectación del sistema nervioso central (SNC), shock séptico o hipoxemia secundaria a enfermedad pulmonar parenquimatosa severa a pesar de la pronta instauración de un tratamiento antibiótico eficaz. El mundo está siendo testigo de una disminución constante en el número de muertes neonatales debido a la sepsis. La mortalidad neonatal por sepsis disminuyó en sólo 28 por ciento ${ }^{4-6}$.
En el continente americano, el $17 \%$ de las muertes neonatales en Sudamérica se atribuyen a la sepsis neonatal, en comparación con solo el $6 \%$ de las muertes neonatales en los países de altos ingresos. La sepsis neonatal, es el principal asesino de recién nacidos en Perú, representa más de un tercio de las muertes neonatales ${ }^{7}$. En la selva peruana, la sepsis neonatal es también una causa importante de morbilidad y de mortalidad neonatal después de la prematurez y asfixia del nacimiento ${ }^{8}$; es responsable del $24 \%$ de las muertes neonatales con una tasa de incidencia tan alta como 10 por cada 1000 nacidos vivos ${ }^{5,7-9}$.

Muchos factores contribuyen a la alta mortalidad debida a las infecciones debido a los retrasos en la identificación y el tratamiento de los recién nacidos con infección, específicamente; incluido el reconocimiento insuficiente de la enfermedad, la demora en la búsqueda de atención a nivel del hogar, el retraso en el inicio del tratamiento y la falta de acceso tanto a los trabajadores de la salud debidamente capacitados como a los servicios de alta calidad para manejar la sepsis. Es particularmente conmovedor que muchas muertes neonatales ocurran en la comunidad, sin que el recién nacido tenga contacto con los servicios de salud apropiados ${ }^{10,11}$.

Las estrategias que pueden prevenir y tratar a los neonatos con sepsis son esenciales para acelerar el progreso de la supervivencia del recién nacido. Sin embargo, en muchos países en desarrollo, la identificación y el tratamiento de los recién nacidos con infección no es satisfactorio y los datos epidemiológicos de los países en desarrollo mostraron diferencias en la incidencia, factores de riesgo, patrones y sensibilidades antimicrobianas de los patógenos y la mortalidad de los países desarrollados. La identificación de los factores de riesgo y la institución temprana de la terapia, pueden mejorar la mortalidad y la morbilidad neonatal ${ }^{5,12}$.

Muchos centros han estudiado los agentes causales comunes de la sepsis neonatal con sus patrones de sensibilidad, pero hay estudios limitados que intentaron verificar los factores de riesgo de la sepsis neonatal en prematuros en el área de estudio y en el país en general; lo mencionado nos motivó a realizar ésta investigación. 
Este estudio, tuvo como objetivo determinar los factores de riesgo asociados a sepsis neonatal temprana en prematuros del Hospital Nacional Docente Madre Niño San Bartolomé: enerodiciembre, 2017.

\section{MÉTODOS}

Se realizó un estudio de casos y controles, observacional, analítico de corte transversal con recolección de datos retrospectiva.

La población estuvo conformada por 210 recién nacidos prematuros $1<37$ semanas de gestación), atendidos en la unidad de neonatología del Hospital Nacional Docente Madre Niño San Bartolomé durante los meses de enero a diciembre del año 2017, a partir de los cuales se calculó el tamaño muestral.

El grupo caso, estuvo conformado por recién nacidos prematuros con sepsis neonatal temprana y el grupo control por recién nacidos prematuros sanos, en ambos grupos los RN fueron atendidos en la unidad de neonatología del Hospital Nacional Docente Madre Niño San Bartolomé, durante los meses de enero a diciembre del año 2017.

El criterio de inclusión fue $\mathrm{RN}$ de parto institucional atendido en el Hospital Nacional Docente Madre Niño San Bartolomé y los criterios de exclusión RN con historias clínicas incompletas y letra ilegible, muertes perinatales y óbitos fetales.

La muestra se calculó a partir de una fórmula para hallar un tamaño muestral para casos y controles tomando en cuenta 2 controles por cada caso (62 casos y 124 controles, ratio 1:2), la prevalencia estimada para la proporción de controles expuestos fue de $6,1 \%$.

La variable dependiente fue la Sepsis neonatal temprana, las variables independientes fueron sexo, peso al nacer, APGAR bajo, edad materna, controles prenatales (CPN), infección del tracto urinario (ITU) en $3^{\circ}$ trimestre, líquido meconial, vía del parto, sufrimiento fetal y ruptura prematura de membranas (RPM). Con una ficha de recolección de datos, diseñada por los autores, se recolectaron los datos de las historias clínicas.

Los datos fueron procesados estadísticamente para poder hallar las asociaciones buscadas. Para la estadística univariada se hallaron las frecuencias y porcentajes en el caso de las variables cualitativas y las medidas de tendencia central y dispersión para las variables cuantitativas. Para el análisis bivariado se hallaron los Odds Ratio con sus respectivos intervalos de confianza al 95\%. Se consideró a los valores $\mathrm{p}<0,05$ como estadísticamente significativos.

Al ser el presente estudio retrospectivo, no se incluyó la participación directa de las pacientes, por tanto, no requirió la firma de un consentimiento informado, además para salvaguardar la confidencialidad no figuró en la ficha de recolección de datos los nombres de las pacientes, lo que permitió la no identificación del mismo. Además, debido a que se trata de un diseño retrospectivo, no existieron riesgos físicos $y / 0$ psicológicos, tampoco potencial invasión de la privacidad, riesgo de muerte y/o alteración de la calidad de vida ni daños a terceros. La custodia sobre la información recogida en la ficha de recolección de datos fue almacenada por un periodo de un año bajo custodia del equipo, siendo posteriormente eliminada.

\section{RESULTADOS}

En el análisis univariado podemos observar las características generales de la población (Tabla 1) y en el análisis bivariado los factores asociados a sepsis neonatal temprana en la población estudiada (Tabla 2). 
Tabla 1. Características generales de los prematuros del Hospital Nacional Docente Madre Niño San Bartolomé en el año 2017.

FRECUENCIA

Sexo

Masculino

Femenino

Peso bajo al nacer

$\mathrm{Si}$

No

Puntaje de APGAR bajo

$\mathrm{Si}$

No

Edad materna

De 35 años a más

Menor de 35 años

Controles prenatales

Menos de 6

6 o más

ITU en el 3er trimestre

Si

No

Liquido meconial

$\mathrm{Si}$

No

Parto por cesárea

$\mathrm{Si}$

No

Sufrimiento fetal

Si

No

Ruptura prematura de membranas

$\mathrm{Si}$

No
61

125

3

183
86

100

39

147

165

19

167

PORCENTAJE

$46,2 \%$

$53,8 \%$

$19,4 \%$

$80,6 \%$

$2,2 \%$

$97,8 \%$

$25,3 \%$

$74,7 \%$

$21,0 \%$

$79,0 \%$

$11,3 \%$

$88,7 \%$

$10,2 \%$

$89,8 \%$

$32,8 \%$

$67,2 \%$

$1,6 \%$

$98,4 \%$

22

$11,8 \%$

164

$88,2 \%$ 


\section{ANÁLISIS BIVARIADO}

Tabla 2. Análisis bivariado de los factores asociados a sepsis neonatal temprana la población estudiada 2017.

\begin{tabular}{|c|c|c|c|c|c|}
\hline \multirow[b]{2}{*}{ Sexo } & \multicolumn{3}{|c|}{ Sepsis neonatal temprana } & \multirow{2}{*}{$\begin{array}{l}\text { ORcrudo } \\
\text { (IC 95\%) }\end{array}$} & \multirow{2}{*}{ Valor p } \\
\hline & Si & No & Total & & \\
\hline Masculino & 26 & 60 & 86 & \multirow{2}{*}{$0,77(0.42-1,42)$} & \multirow{2}{*}{0,438} \\
\hline Femenino & 36 & 64 & 100 & & \\
\hline \multicolumn{6}{|l|}{ Peso bajo al nacer } \\
\hline $\mathrm{Si}$ & 21 & 15 & 36 & \multirow{2}{*}{$3,72(1,75-7,91)$} & \multirow{2}{*}{0,001} \\
\hline No & 41 & 109 & 150 & & \\
\hline \multicolumn{6}{|l|}{ Puntaje de APGAR bajo } \\
\hline $\mathrm{Si}$ & 3 & 1 & 4 & \multirow{2}{*}{$6,25(0,64-61,41)$} & \multirow{2}{*}{0,109} \\
\hline No & 59 & 123 & 182 & & \\
\hline \multicolumn{6}{|l|}{ Edad materna } \\
\hline De 35 años a más & 23 & 24 & 47 & \multirow{2}{*}{$2,45(1,24-4,85)$} & \multirow{2}{*}{0,012} \\
\hline Menor de 35 años & 39 & 100 & 139 & & \\
\hline \multicolumn{6}{|l|}{ Controles prenatales } \\
\hline Menos de 6 & 21 & 18 & 39 & \multirow{2}{*}{$3,02(1,46-6,23)$} & \multirow{2}{*}{0,004} \\
\hline 6 o más & 41 & 106 & 147 & & \\
\hline \multicolumn{6}{|l|}{ ITU en el 3er trimestre } \\
\hline $\mathrm{Si}$ & 12 & 9 & 21 & \multirow{2}{*}{$3,07(1,21-7,74)$} & \multirow{2}{*}{0,025} \\
\hline No & 50 & 115 & 165 & & \\
\hline Liquido meconial & 62 & 124 & 186 & & \\
\hline $\mathrm{Si}$ & 12 & 7 & 19 & \multirow{2}{*}{$4,01(1,49-10,78)$} & \multirow{2}{*}{0,008} \\
\hline No & 50 & 117 & 167 & & \\
\hline Parto por cesárea & 62 & 124 & 186 & & \\
\hline Si & 20 & 41 & 61 & \multirow{2}{*}{$0,964(0,50-1,84)$} & \multirow{2}{*}{0,999} \\
\hline No & 42 & 83 & 125 & & \\
\hline Sufrimiento fetal & 62 & 124 & 186 & & \\
\hline $\mathrm{Si}$ & 2 & 1 & 3 & \multirow{2}{*}{$4,1 \quad(0,36-46,11)$} & \multirow{2}{*}{0,258} \\
\hline No & 60 & 123 & 183 & & \\
\hline Ruptura prematura de membranas & 62 & 124 & 186 & & \\
\hline Si & 12 & 10 & 22 & \multirow{2}{*}{$2,74(1,11-6,75)$} & \multirow{2}{*}{0,031} \\
\hline No & 50 & 114 & 164 & & \\
\hline TOTAL & 62 & 124 & 186 & & \\
\hline
\end{tabular}

En la tabla 3 podemos apreciar el análisis multivariado de los factores que salieron asociados en el análisis bivariado, se conserva la significancia de todos.

ANÁLISIS MULTIVARIADO

Tabla 3. Análisis multivariado de los factores asociados a sepsis neonatal temprana en neonatos prematuros del Hospital Nacional Docente Madre Niño San Bartolomé en el año 2017.

\begin{tabular}{lcccc} 
& OR Ajustado & \multicolumn{2}{c}{ Intervalo de confianza al 95 \% } & Valor P \\
& 4,031 & 1,743 & 9,318 & 0,001 \\
\hline Bajo peso al nacer & 2,729 & 1,266 & 5,880 & 0,010 \\
Edad de 35 a más & 3,315 & 1,452 & 7,567 & 0,004 \\
Menos de 6 controles prenatales & 2,947 & 1,063 & 8,174 & 0,038 \\
ITU en el 3er trimestre & 5,822 & 1,920 & 17,653 & 0,002 \\
Líquido meconial & 2,789 & 1,035 & 7,511 & 0,042 \\
Ruptura prematura de membranas & & & & \\
\hline
\end{tabular}




\section{DISCUSIÓN}

Los resultados de este estudio muestran que existe una relación entre bajo peso al nacer y la presencia de sepsis neonatal temprana; esto podría explicarse por la disfunción inmune y la ausencia de anticuerpos IgG maternos adquiridos de forma transplacentaria, debido al poco desarrollo de los neonatos.

Otros estudios tienen resultados similares como el desarrollado en Bélgica y publicado en el año 2015, donde se trabajó con 342 neonatos que sobrevivieron las primeras 72 horas después del nacimiento, y que fueron hospitalizados en la Unidad de Cuidados Críticos, se encontró que el riesgo de sepsis neonatal temprana aumenta conforme disminuye el peso al nacer ${ }^{15}$.

En un estudio realizado en el Hospital Nacional Sergio E. Bernales de Lima durante el año 2016, reportan que un peso menor a $2,5 \mathrm{Kg}$ al nacer es un factor de riesgo para sepsis neonatal temprana ${ }^{16}$.

También en el Hospital de lquitos, encontraron que un peso muy bajo al nacer tuvo mayor proporción de sepsis neonatal que los controles sin sepsis neonatal ${ }^{17}$ En otro estudio realizado en Paita, encuentran que tener un peso entre 1500 y 2499 gramos es un factor de riesgo para sepsis neonatal temprana. Además, reportan que la edad materna mayor a 35 años de edad es un factor de riesgo para sepsis neonatal temprana, con un OR ajustado de 2,729 probablemente debido al aumento del riesgo obstétrico ${ }^{18}$. Un resultado similar fue reportado en el estudio realizado en el Hospital Nacional Sergio E. Bernales de Lima, donde el riesgo de sepsis neonatal temprana aumenta en el grupo de madres en edad de riesgo (gestantes de más de 35 años) y también en gestantes adolescentes, pues presentan un mayor riesgo obstétrico ${ }^{16}$.

Otro factor que resultó ser significativo estadísticamente fue el número de controles prenatales, como se sabe, la Organización Mundial de la Salud recomienda un mínimo de 6 controles prenatales para asegurar un curso saludable de la gestación, prevenir cualquier tipo de complicación y detectar otros factores de riesgo para morbilidad materno perinatal; una cantidad inadecuada de controles prenatales, podría poner en riesgo el curso del embarazo. Este resultado concuerda con el encontrado en el Hospital las Mercedes de Paita donde uno de los factores de riesgo para sepsis neonatal temprana fue tener menos de 6 controles prenatales ${ }^{18}$. Lo mismo fue reportado en el Hospital Nacional Dos de Mayo, donde se trabajó con 32 casos y 64 controles y el tener menos de 6 controles prenatales se asoció con sepsis neonatal temprana ${ }^{19}$. En el Hospital Iquitos también reportaron que menos de 6 controles prenatales en las gestantes está relacionado con mayor prevalencia de sepsis neonatal temprana que en las mujeres con 6 controles prenatales o más ${ }^{17}$, resultado similar fue encontrado en un estudio realizado en el Hospital Nacional Sergio E. Bernales de Lima ${ }^{16}$.

Se encontró en nuestro estudio que la infección urinaria en el 3er trimestre fue un factor de riesgo para sepsis neonatal temprana. Esto podría explicarse debido a que las infecciones en el último trimestre del embarazo pueden facilitar la contaminación del canal de parto y la exposición del recién nacido a las bacterias responsables de la infección urinaria aumentando el riesgo de infecciones tempranas. Resultados similares fueron encontrados en los estudios realizados en los Hospitales, Nacional Sergio E. Bernales de Lima y Las Mercedes de Paita, donde la infección del tracto urinario en el último trimestre se asoció positivamente con la sepsis neonatal temprana ${ }^{16,18}$. Otro factor de riesgo asociado a sepsis neonatal temprana fue la presencia de líquido amniótico meconial. El meconio se acumula en el tracto gastrointestinal fetal durante el tercer trimestre del embarazo y es el primer flujo intestinal que se libera dentro de las primeras 48 horas después del nacimiento. Cabe destacar que, dado que el meconio y todo el contenido del tracto gastrointestinal está ubicado "de manera extracorpórea", sus componentes están ocultos y normalmente el sistema inmunitario fetal no los reconoce. Estas sustancias pueden ser excretadas de forma temprana en caso de que el RN pase por algún sufrimiento fetal, como puede ser consecuencia de alguna infección temprana.

En un estudio realizado en el Hospital Nacional Arzobispo Loayza, se encontró que la presencia de líquido amniótico meconial presenta una asociación con la sepsis neonatal temprana en recién nacidos prematuros ${ }^{20}$.

Lo mismo encontramos nosotras, donde el líquido amniótico meconial resultó ser un factor de riesgo para sepsis neonatal temprana. En la India reportaron que el líquido meconial fluido es un indicador de sufrimiento fetal y que podría ser un indicador temprano de infección por estreptococo ${ }^{21}$.

Por último, la ruptura prematura de membranas 
también resultó ser un factor de riesgo para sepsis neonatal temprana. Esto se debe a que las membranas amnióticas trabajan como protectoras de bacterias contaminantes del canal de parto, y al estar rotas de manera prematura antes del parto, pueden permitir la invasión de bacterias del medio ambiente con la posible infección del canal de parto, aumentando el riesgo de infección de los recién nacidos por aumentar la exposición a estos gérmenes. Un estudio realizado en Estados Unidos encontró que a mayor tiempo que transcurre con las membranas rotas aumenta el riesgo de sepsis neonatal temprana ${ }^{22}$. Otro estudio realizado en Hospital Iquitos, respalda estos resultados ${ }^{17}$.

\section{CONCLUSIÓN}

Existe asociación entre el bajo peso al nacer los controles prenatales, la infección del tracto urinario en el 3er trimestre del embarazo, la edad materna, el líquido meconial y la aparición de sepsis neonatal temprana en prematuros del Hospital Nacional Docente Madre Niño San Bartolomé en el año 2017.

\section{REFERENCIAS BIBLIOGRÁFICAS}

1. Singer M, Deutschman C, Seymour CW, ShankarHari M, Annane D, Bauer M, et al. The Third International Consensus Definitions for Sepsis and Septic Shock. JAMA. 2016 Feb 23; 315(8):801-10.

2. Ferrer Montoya R, Rodríguez de la Fuente F, Estévez Llovet MC, Cuesta García Y, Licea Gómez R. Factores de riesgo materno de la infección de debut precoz en neonatos pretérminos. Multimed [Internet]. 2011 Abr-Jun 15(2):18-32. Disponible en: http:// www.multimedgrm.sld.cu/articulos/2011/v15-2/5.html.

3. Haque KN. Defining common infections in children and neonates. J Hosp Infect. junio de 2007;65 Suppl 2:110-4.

4. WHO | Every Newborn: an action plan to end preventable deaths [Internet]. WHO. [citado 11 de julio de 2018]. Disponible en: http:// www.who.int/maternal_child_adolescent/documents/every-newbornaction-plan/en/

5. Committing to Child Survival: A Promise Renewed - Progress Report 2015 [Internet]. UNICEF. [citado 11 de julio de 2018]. Disponible en: https://www.unicef.org/publications/index_83078.html

6. Chu S-M, Hsu J-F, Lee C-W, Lien R, Huang H-R, Chiang M-C, et al. Neurological Complications after Neonatal Bacteremia: The Clinical Characteristics, Risk Factors, and Outcomes. PLoS ONE [Internet]. 3 de noviembre de 2014 [citado 11 de julio de 2018];9(11). Disponible en: https://www.ncbi.nlm.nih.gov/pmc/articles/PMC4217713/

7. UNICEF. UNICEF - El objetivo: mejorar la salud materna [Internet]. 2015 [citado 28 de abril de 2017]. Disponible en: https://www.unicef. org/spanish/mdg/maternal.html

8. Berhanu DD. Community Based Newborn Care Baseline Survey Report Ethiopia, October 2014 [Internet]. 2014 [citado 11 de julio de 2018]. Disponible en: https://ideas.Ishtm.ac.uk/report/communitybased-newborn-care-baseline-survey-report-ethiopia-october-2014/

9. Welay A, Tadesse K, G / Tsadik T. Process Evaluation of Health Management Information System Implementation Status in Public Health Facilities of Mekelle Zone, Tigray, Ethiopia. Res Rev J Comput Biol. 1 de enero de 2017:6:36-49.
Contribuciones de autoría: Las autoras participaron en la generación, redacción y aprobación final d el artículo original.

\section{Financiamiento: Autofinanciado.}

Conflicto d e i nterés: $L$ as a utoras d eclaran $n$ o t ener conflicto de intereses en la publicación de este artículo.

Recibido: 02 de diciembre 2018

Aprobado: 30 de mayo 2019

Correspondencia: Gretzelle Burga Montoya.

Dirección: Centro de Salud Pichanaki, Pichanaki, Chanchamayo, Junín-Perú. Teléfono: +1974639950

Correo:grety_15_12@hotmail.com
10. Edmond K, Zaidi A. New approaches to preventing, diagnosing, and treating neonatal sepsis. PLoS Med. 9 de marzo de 2010;7(3):e1000213.

11. Qazi SA, Stoll BJ. Neonatal Sepsis: A Major Global Public Health Challenge. Pediatr Infect Dis J. enero de 2009;28(1):S1.

12. GA A, Belay WL, Moges F, Moges B, Anagaw B, Yismaw G, et al. Bacterial profile and drug susceptibility pattern of neonatal sepsis in Gondar University Hospital, Gondar northwest Ethiopia. Pharma Lett. 1 de enero de 2012;4:1811-6.

13. Heron M. Deaths: Leading Causes for 2013. Natl Vital Stat Rep Cent Dis Control Prev Natl Cent Health Stat Natl Vital Stat Syst. 16 de febrero de 2016;65(2):1-95.

14. Julca Ramírez EM. Factores de riesgo asociados a sepsis neonatal temprana en el hospital nacional P.N.P. Luis N. Sáenz en el periodo de enero del 2016 a setiembre del 2017 [Internet] [Tesis de grado]. [Lima, Perú]: Universidad Ricardo Palma.; 2018 [citado 16 de febrero de 2019]. Disponible en: http://repositorio.urp.edu.pe/handle/URP/1163

15. Hilde Verstraete E, De Coen K, Vogelaers D, Blot S. Risk Factors for Health Care-Associated Sepsis in Critically III Neonates Stratified by Birth Weight. Pediatr Infect Dis J. noviembre de 2015;34(11):1180.

16. Sandoval Robles SL. Factores de riesgo asociados a Sepsis Neonata Temprana en el Hospital Nacional Sergio E. Bernales de Lima, EneroDiciembre 2016. Univ Nac Federico Villarreal [Internet]. 2018 [citado 11 de julio de 2018]; Disponible en: http://repositorio.unfv.edu.pe/handle/ UNFV/1755

17. Ampuero Túnjar GC. Factores de riesgo asociados a mortalidad en sepsis neonatal temprana en el Hospital lquitos César Garayar García del año 2010 - 2013. Univ Nac Amaz Peru [Internet]. 2016 [citado 11 de julio de 2018]; Disponible en: http://repositorio.unapiquitos.edu.pe/ handle/UNAP/4024

18. Palacios Bruno JJ. Factores de riesgo para sepsis neonatal precoz en prematuros del Hospital las Mercedes de Paita de enero a diciembre del 
2017. Univ Nac Piura UNP [Internet]. 2018 [citado 11 de julio de 2018]; Disponible en: http://repositorio.unp.edu.pe/handle/UNP/1193

19. lyo Alberti FL. Controles Prenatales y Puntaje de Apgar menor de 7 como Determinantes de Sepsis Neonatal Temprana. Hospital Nacional Dos de Mayo. 2014. Univ Priv Antenor Orrego - UPAO [Internet]. 2015 [citado 11 de julio de 2018]; Disponible en: http://repositorio.upao.edu. pe/handle/upaorep/1590

20. Espiritu Falcon LA. Factores Asociados a la Sepsis Neonatal Temprana en el Servicio de Neonatología del Hospital Nacional Arzobispo Loayza en el año 2017. Univ Nac Federico Villarreal [Internet]. 2018 [citado 11 de julio de 2018]; Disponible en: http://repositorio.unfv.edu.pe/handle/ UNFV/1705
21. Santhanam S, Arun S, Rebekah G, Ponmudi NJ, Chandran J, Jose R, et al. Perinatal Risk Factors for Neonatal Early-onset Group B Streptococcal Sepsis after Initiation of Risk-based Maternal Intrapartum Antibiotic Prophylaxis-A Case Control Study. J Trop Pediatr [Internet]. [citado 11 de julio de 2018]; Disponible en: https://academic.oup.com/tropej/ advance-article/doi/10.1093/tropej/fmx068/4210260

22. Drassinower D, Friedman AM, Običan SG, Levin H, GyamfiBannerman $C$. Prolonged latency of preterm premature rupture of membranes and risk of neonatal sepsis. Am J Obstet Gynecol. 1 de junio de 2016;214(6):743.e1-743.e6.
Indizado en:

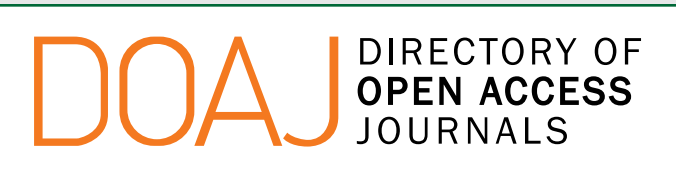

https://doaj.org/

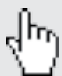

Revista de la

Facultad de

Medicina Humana

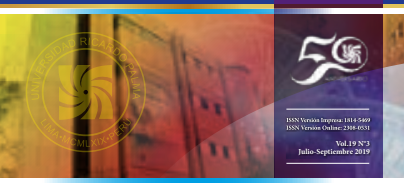

(2) amsoben

DOAJ 